\title{
ETHISCHE KOMPETENZENTWICKLUNG IN DER POLIZEIAUSBILDUNG
}

\author{
Dr. Giedrè Paurienè \\ Akademie der öffentlichen Sicherheit der Mykolas-Romeris-Universität
}

\begin{abstract}
Annotation. In diesem Artikel wird die Entwicklung der ethischen Kompetenz eines Polizisten in einer modernen Hochschule diskutiert. Die Polizei repräsentiert die Autorität des Staates in der Kommunikation mit den Bürgern. Da Polizisten sich mit Konflikten auf verschiedenen Ebenen der Gesellschaft auseinandersetzen müssen, sollten ethische Dilemma-fähigkeiten Teil der beruflichen Kompetenz werden. Daher ist die Entwicklung der ethischen Kompetenz als Bestandteil der polizeilichen Berufskompetenz als ein wichtiger Moment in der Ausbildung von Polizeibeamten zu betrachten. Im Mittelpunkt dieser Artikel steht die ethische Kompetenzstruktur von Polizeibeamten. Es werden die professionelle Deontologie und die wichtigste Kategorie des deontologischen Intuitionismus - Verpflichtung betont. Man kommt zu Schlussfolgerungen, dass der Prozess der ethischen Kompetenzentwicklung von den Polizeibeamten aus einer systematischen Reflexion der beruflichen moralischen Anforderungen bestehen muss, um ethische Kompetenz zu reflektieren und sie mit anderen Erfahrungen zu vergleichen.
\end{abstract}

Schlüsselwörter: ethische Kompetenz, professionelle Deontologie, Verpflichtung, Kompetenzentwicklung, Reflexion, Polizeiausbildung.

\section{Einleitung}

Die Anforderungen, auf die sich die Polizei professionell einstellen muss, ergeben sich aus verschiedenen Situationen, in denen die Polizei handeln muss. Unsere Zeit wird auf viele Arten genannt: "Risikogesellschaft" (Beck, 1992), Epoche der Unempfindlichkeit oder "der Konsum von Menschenleben" (Baumann, 2010). Die Beziehungen zwischen den Gesellschaftsmitgliedern werden immer wieder komplizierter, sie werden durch den sich beschleunigenden Wandel, durch die Herausforderungen der Globalisierung und der sich schnell entwickelnden neuen Technologien beeinflusst. Smalskys (2008) analysierte Kompetenzen- und Qualifikationsentwicklungsfragen der litauischen Polizei. Er behauptete, dass die Ausbildung der Polizei als Konzept „Polizeitätigkeit als soziale Dienstleistung für Bürger“ ausgerichtet sein kann und sollte.

Vertreter des Polizeiberufes müssen sich an Gesetze und ethische Verpflichtungen halten. Im Beruf des Polizeibeamten sollten moralische und ethische Fragen 
$\mathrm{zu}$ einem wesentlichen Bestandteil werden, weil gesellschaftlich immer wieder kritisch auf die polizeiliche Berufsethik gesehen wird, und in der medialen Öffentlichkeit wird von Tag zu Tag das Thema des polizeilichen Dienstes besprochen.

Einzelne Berufe haben ihre eigene Kultur, ihr Denken, Verhalten (Palidauskaite, 2007) und jeder Beruf definiert Kompetenz anders. Zusammenfassend kann Kompetenz als ein streng spezialisiertes System von Können, Erfahrungen und Fähigkeiten interpretiert werden, das zur Erreichung der Ziele einer bestimmten spezialisierten Tätigkeit erforderlich ist (Weinert, 2001).

Bei der Diskussion über die Struktur der professionellen Kompetenz unterscheiden die Autoren auch die Komponenten dieser Kompetenz. Laužackas (1999) identifiziert die fachlichen, methodischen und sozialen Kompetenzen als Teil der beruflichen Kompetenz. Ihm zufolge ist professionelles Wissen oder fachliche Kompetenz eine der wichtigsten. Es ist jedoch immer unklar, was der Inhalt des „relevanten, aktuellen Wissens“ (Yelloly, 1995, zit. nach Gapšytė \& Snieškienė, 2015) im Beruf des Polizeibeamten ist. Gapšytė und Snieškienė (2015), basierend auf Wissenschaftler Arduino (2006), unterteilen die Komponenten der professionellen Kompetenz folgend: kognitive Kompetenz beinhaltet formelles und implizites Wissen basierend auf Erfahrung; funktionale Kompetenz besteht aus Fähigkeiten und technischem Wissen; persönliche Kompetenz ist das Wissen, wie man sich in einer bestimmten Situation verhält; ethische Kompetenz basiert auf professionellen und persönlichen Werten.

Was die Bedeutung von Werten anbelangt, ist man am besten in der Lage, Kompetenzen nur dann auszudrücken, wenn die Umstände seinen Werten entsprechen. Wie Psychologen Hautzinger und Pössel (2017) behaupten, psychisches Wohlbefinden und Energie wird entscheidend davon gespeist, dass ein Individuum sein Leben an Werten ausrichtet, seine Werte frei wählt und sein alltägliches Handeln daran ausrichtet. Werte geben die Richtung vor, doch sind unerreichbar, denn man kann immer weitergehen. Werte und Wertorientierung, nach den oben genannten Psychologen, entsprechen in weiten Teilen den Konstrukten wie Grundüberzeugungen, Einstellungen, Pläne, Schemata und sind positiv formulierte Haltungen, selbstbezogene Orientierungen, wodurch sie deutlich energetischer, motivierender und handlungsbestimmend sind.

Die Polizeiarbeit kann aufgrund der besonderen Befugnisse der Polizei, der vertraulichen Informationen, die ihr zur Verfügung stehen, leicht zu unethischem Verhalten führen Die Polizeiarbeit sieht sich ständig Verbrechen gegenüber. Man muss auch Diskretionsrecht im Hinterkopf behalten, d.h. das Recht eines öffentlichen Organs, zwischen mehreren rechtlich zulässigen Möglichkeiten nach eigenem Ermessen zu entscheiden. Bei der Polizeiausbildung ist es deshalb notwendig, wie man CEPOL Common Curriculum - Police Ethics and Prevention of Corruption (2005) betont, die Bereiche zu diskutieren, in denen das Risiko von unethischem Verhalten und die Versuchung der Korruption besteht. Die zukünftigen Polizisten sollten ermutigt werden, die Werte und modernen Praktiken, Strukturen und Syste- 
me zu analysieren, Schwächen, Gesetzeslücken, Privilegien durch Bekanntschaften zu identifizieren und usw. Daher sind die Entwicklung und Erforschung ethischer Kompetenz in diesem Bereich relevant.

Es ist wichtig, Antworten auf die Frage zu finden, was der Inhalt der ethischen Kompetenz von den Polizeibeamten ist und wie sollte man diesen Inhalt den Studierenden auf beste Art und Weise vermitteln?

Ziel dieses Artikels ist es, die Möglichkeiten der ethischen Kompetenzentwicklung in der Polizeiausbildung zu besprechen.

Zum Ziel der Untersuchung führen folgende Aufgaben:

1. basierend auf professioneller Deontologie die ethische Kompetenzstruktur von den Polizeibeamten darzustellen;

2. den Ausbildungsprozess der ethischen Kompetenz bei den Polizeibeamten zu besprechen.

Gegenstand der vorliegenden Untersuchung ist die ethische Kompetenzentwicklung in der Polizeiausbildung.

Untersuchungsmethoden der vorliegenden Arbeit: theoretische Methoden - vergleichende und verallgemeinernde Methoden, die für die Analyse der wissenschaftlichen Literatur benutzt wurden.

\section{Ethische Kompetenzstruktur von den Polizeibeamten}

Der Begriff „ethische Kompetenz“ wird nach Ansicht Richter, Henkens und Ritt (2011) in der Praxis oft unscharf verwendet. Unter Berücksichtigung des Konzepts der „moralischen Verpflichtung“, kann „ethische Kompetenz“ genauer differenziert werden. Wie Rutkowsky (2017) bemerkt, umgangssprachlich werden Moral und Ethik immer öfter gleichbedeutend gebraucht, aber seiner Meinung nach, beinhaltet Moral die Normen und Regeln, die das Zusammenleben regeln. Und die Ethik hat Ziel, „die Gültigkeit moralischer Regeln zu prüfen und auf diese Weise selber handlungsorientierend zu sein" (Rutkowsky, 2017, S. 19). Daher sind vor allem die normative und die angewandte Ethik, denen es um moralische Fragen z. B. im Polizeiberuf geht, von der Moral nur noch insofern zu unterscheiden, als sie ihre Voraussetzungen und Schlussfolgerungen besonders sorgfältig erwägen.

Bei der Definition der ethischen Kompetenz von Polizeibeamten ist es zweckmäßig sich Rabe (2005) anzuschließen. Rabe (2005) gibt eine inhaltreiche Definition: „ethische Kompetenz beinhaltet die Fähigkeit zur Reflexion, Formulierung und Begründung der eigenen moralischen Orientierungen, weiter die Fähigkeit zum Erkennen moralischer Probleme in der eigenen Praxis, Urteilsfähigkeit, Diskursfähigkeit und schließlich Wachheit und Mut, auch tatsächlich moralisch zu handeln" (Rabe, 2005, S. 131). In ihrer Definition fasste Rabe (2005) der ethischen Kompetenz alle Merkmale der zu Beginn erarbeiteten Begriffe von Laužackas (1999) auf und gibt so gleichermaßen Fähigkeiten vor, woran der Ethikunterricht ausgerichtet werden kann. Sicherlich soll der Ethikunterricht die genannten Kompetenzen, wie 
fachliche Kompetenz, soziale Kompetenz, methodische Kompetenz fördern und entwickeln, aber nicht zu vergessen sei die persönliche Kompetenz. Doch für den Ethikunterricht hält Rabe (2005) den Begriff der ethischen Kompetenz als eine geeignetere Zielorientierung.

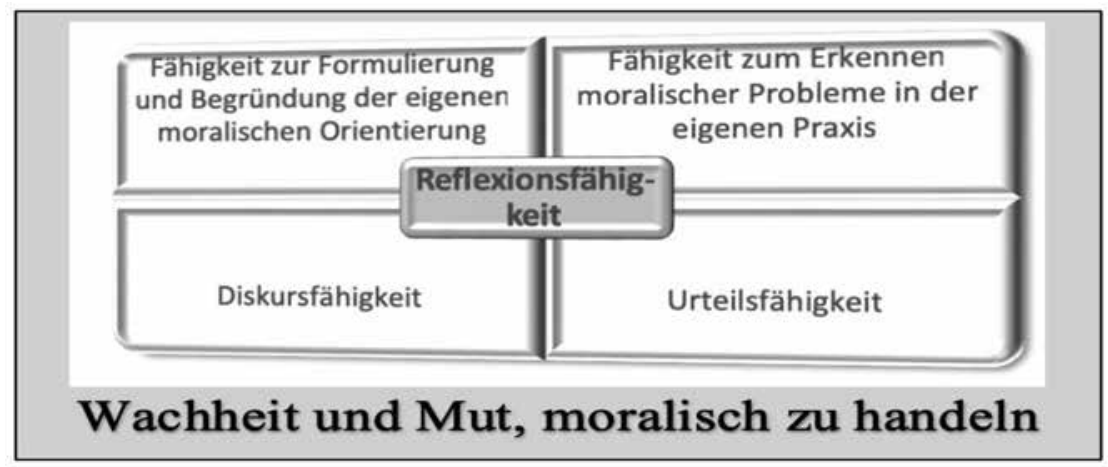

Bild 1. Ethische Kompetenzstruktur der Polizeibeamten.

Auf dem Grund der ethischen Kompetenzstruktur der Polizeibeamten liegen zwei persönliche Werte - Wachheit und Mut. Moralische Werte, weist Rutkowsky (2017) hin, bezeichnen ein Wollen, Normen ein Sollen. Dies klingt zunächst wie ein Gegensatz, tatsächlich aber sind Normen und Werte zwei Seiten derselben Medaille, denn jede moralische Norm enthält einen Wert, sonst hätte sie keinen Sinn. Normen sind Werte, die von einer Gemeinschaft als verbindlich angesehen werden und deshalb für alle ihre Mitglieder gelten sollen. In diesem Fall geht die rede über die Norm - moralisch zu handeln.

Es gibt Situationen im Leben, in denen eine bestimmte Art der Kommunikation erforderlich ist, die nicht von unseren Überzeugungen abhängt, sondern von dem Diktat der sozialen Rolle, die wir haben. Die Verwirklichung einer sozialen Rolle ist ein zentrales Thema in der Berufsethik. Die Besonderheit der Berufsethik ist, dass es nicht genug gute Absichten, Willen und besondere Kenntnisse, Mitgefühl und Können gibt. Wie behauptet Laurinavičius (2001), im Polizeiberuf sollte reglementiertes Verhalten mit Menschen gelten, ohne zu beachten, ob diese Person eines solchen Verhaltens würdig ist, d.h. ohne ihm Sympathie oder Antipathie zu zeigen.

Der Beruf des Polizeibeamten hat neben den allgemeinen ethischen Anforderungen viele zusätzliche Anforderungen, die durch ihre soziale Rolle bedingt werden. Der Begriff der moralischen Pflicht ist wesentlich auf den Begriff der moralischen Forderung oder Erwartung bezogen, die ihrerseits nicht alleine stehen, sondern in einen weiter ausgreifenden Kontext ethischer Begriffe einbezogen sind, behauptet Brandhorst (2013). Wenn der Polizeibeamte die berufsethische Anforderungen missachtet, macht er seinen Job bedeutungslos, erfüllt seine Aufgaben nicht und kann daher als unverantwortlicher Mensch betrachtet werden. Der Polizeibe- 
amte muss die ihm übertragenen Aufgaben unter Beachtung der pflichtigen Anforderungen unabhängig von seiner Stimmung oder zufälligen Umständen erfüllen. Verantwortung, wie Laurinavičius (2001) sagt, ist eine Kategorie von Ethik und Recht, die die Beziehung einer bestimmten Person zur Öffentlichkeit aufzeigt. Die moralische Verantwortung ist ein bestimmter Aspekt der Beziehung der Menschen zur Welt, eine Möglichkeit, die Aktivitäten und das Verhalten der Persönlichkeit zu regulieren. Moralische Verantwortung geht „von der sozialen Institution der Moral aus, die eine der Formen des sozialen Bewusstseins ist, die das menschliche Verhalten in allen Bereichen des öffentlichen Lebens reguliert. Im polizeilichen Dienst kann moralische Verantwortung sowohl individuell als auch kollektiv sein." (Laurinavičius 2001, S. 41).

Kategorie der moralischen Verantwortung steht nebenbei der Kategorie der Verpflichtung - der wichtigsten Kategorie des deontologischen Intuitionismus. Der Begriff der moralischen Pflicht enthält den Begriff der Moral und ist zugleich die begriffliche Grundlage einer Verpflichtung. Eine moralische Berufsverpflichtung ist keine Pflicht, die ein Polizeibeamter beim Dienst leisten muss, sondern ein Gewissensbefehl. Infolgedessen erfüllt ein ehrlicher Beamter seine Pflichten, indem er die innere Notwendigkeit spürt. Die Verpflichtung ist eng mit der moralischen Berufsverantwortung verbunden: Die Erfüllung der Verpflichtung setzt die Verantwortung des Beamten voraus, und die Verantwortung erfordert die Erfüllung der Verpflichtung. Die Verpflichtung macht eine Person zur Verantwortung, und die moralische Verantwortung verpflichtet.

Eine Pflichtethik oder deontologische Ethik (von gr. deon - Pflicht) lässt die Folgen eines Tuns zwar nicht gänzlich außer Acht, aber das moralische Urteil fällt über die Absicht des Handelnden (Rutkowsky, 2017). Entscheidend ist, ob diese den moralischen Pflichten entspricht. Wie bereits erwähnt, die Hauptkategorie des deontologischen Intuitionismus sind moralische Verpflichtung. Das Wort „muss“ gilt nur für Handlungen. Die Pflichten, obwohl offensichtlich sind, werden doch intuitiv gefühlt, und basieren sich auf persönlichen Werten, Überzeugungen, Einstellungen.

\section{Ausbildungsprozess der ethischen Kompetenz bei den Polizeibeamten}

In den Definitionen der Ethik wird die Reflexion als die bestimmende Fähigkeit benannt. Diese Fähigkeit sollte ein generelles Ziel der Polizeiausbildung sein, denn nicht nur für den Erwerb einer ethischen Kompetenz ist die Reflexionsfähigkeit von Bedeutung, sondern für die Arbeit als professionelle Berufsgruppe und für die Umsetzung des Schützen-Verteidigen-Helfen-prozesses ist diese Fähigkeit ebenso wichtig. Die Reflexionskompetenz der zukünftigen Polizeibeamten und die Entwicklung ihrer Fähigkeiten und Kompetenzen in der Hochschulbildung sind eine der effektivsten Formen der beruflichen Weiterentwicklung, ein wichtiges Ziel für die Ausbildung von zukünftigen Fachkräften, da es möglich ist, sich in der Ana- 
lyse und Reflexion ihrer Aktivitäten weiterhin effektiv auszubilden und zu trainieren (Correia \& Bleicher, 2008). Es ist wichtig, Bedingungen für die Entwicklung von Reflexionskompetenzen zu schaffen, die die wesentlichen Elemente - Persönlichkeitserfahrungen, Denken, Emotionen, Handlungen und Werte im sozialen und politischen Kontext, in dem der Lernende lebt, umfassen.

Die Wahrnehmung der Reflexion in der Praxis beginnt mit einem Verständnis dessen, was man tut, und endet mit der kritischen Würdigung der Ideologie und des Glaubens, die dem Denken und Handlungsgefühl zugrunde liegen (Yip, 2006, zit. nach Gapšytė \& Snieškienè, 2015). Sich seiner eigenen Wertvorstellung bewusst zu sein und diese auch benennen zu können, ist ein wichtiger Aspekt, wenn es um die Betrachtung ethischer Problemsituationen geht. Die Fähigkeit zur Formulierung und Begründung der eigenen moralischen Orientierung kann behilflich sein, um alternative Antworten auf bestimmte Fragen und mögliche ethische Problemlösungen zu finden und so die Feinheiten der beruflichen Tätigkeit leichter zu verstehen.

Die Fähigkeit zum Erkennen moralischer Probleme in der eigenen Praxis baut auf den zuvor benannten Fähigkeiten und der parallel erworbenen fachlichen und erfahrenen praktischen Ausbildung auf. Hier werden die persönlichen Wertvorstellungen in Beziehung zu den ethischen Ansprüchen der Berufsgruppe und dem Erlebten in der Praxis gebracht. Ein aktives Bestreben in einem dynamischen Praxisumfeld allgemeines Wissen über sich und den Beruf zu generieren, gehört, nach Lam, Wong und Leung (2007), zu den Reflexionsfähigkeiten.

Diskursfähigkeit unterliegt damit hohen Anforderungen, sie muss erlernt und eingeübt werden. Eine Reihe unterschiedlicher Kompetenzen muss erworben werden: Sachkompetenz in der zu verhandelnden Streitfrage, sozial-kommunikative Kompetenzen u.a. Dieser Kompetenzbereich ist nicht als ein in sich geschlossener Gegenstand erlernbar. Zur Urteilsfähigkeit gehören ethische Urteilskompetenzen. Im Alltag finden Handlungsentscheidungen häufig intuitiv; wie Wagener und Schiewek (2015) behaupten, die einzelnen Schritte, wie man zu einem (moralischen) Urteil und zur Entscheidung kommt, sind meist nicht bewusst. Verfahren ethischer Urteilsfindung machen diesen Prozess transparent. Sie können helfen, das eigene Handeln so zu analysieren und zu bewerten, dass verschiedene Handlungsmöglichkeiten systematisch erkannt und angemessen bewertet werden können (Wagener, Schiewek, 2015, S.199). Kritisches Denken und Fähigkeiten zur Problemlösung sind entscheidend für die Verbesserung der Reflexionskompetenz, für ein effektiveres Engagement (Lay, McGuire 2010) und für Ausbildungsprozess der ethischen Kompetenz bei den Polizeibeamten. Verallgemeinert lässt sich sagen, dass der Prozess der ethischen Kompetenzentwicklung von den Polizeibeamten aus einer systematischen Reflexion der beruflichen moralischen Anforderungen bestehen muss, um ethische Kompetenz zu reflektieren und sie mit anderen Erfahrungen zu vergleichen. 


\section{Schlussfolgerungen}

Die ethische Kompetenzstruktur der Polizeibeamten ist durch Komplexität gekennzeichnet und spiegelt Fähigkeiten und persönliche Werte wider, aber ihre theoretische Trennung ist einfacher zu handhaben als praktische. Die in diesem Artikel dargestellte Struktur der ethischen Kompetenz ist bedingt, zeigt jedoch die Schlüsselbereiche, die für die Entwicklung von Fähigkeiten und Kompetenzen relevant sind, die die ethische Kompetenz der Polizeibeamten umfassen. Basierend auf professioneller Deontologie im Sinne der Verpflichtung - moralisch zu handeln, zeigt die Struktur der ethische Kompetenzstruktur von Polizeibeamten durch bestimmende Reflexionsfähigkeit sowie Fähigkeit zur Formulierung und Begründung der eigenen moralischen Orientierung, Fähigkeit zum Erkennen moralischer Probleme in der eigenen Praxis sowie Diskursfähigkeit und Urteilsfähigkeit.

Für das Verhalten in moralisch relevanten Situationen sind Verhaltensspezifische Einstellung, kontextbezogene subjektive Norm und wahrgenommene Verhaltenskontrolle wichtig. Die ethische Kompetenz ist wichtig für den Unterricht von Studierenden der Polizeitätigkeit. Sie bietet die Möglichkeit, einen reflektierten, kompetenten Polizeibeamten auszubilden, der in definierten und unsicheren beruflichen Situationen agieren kann.

\section{Literatur}

1. Bauman, Z. (2010). Slapčiausia vartotojų visuomenės paslaptis. Iš Vartojamas gyvenimas. Vilnius: Apostrofa academia.

2. Beck, U. (1992). Risk Society: Towards a New Modernity (Vol. 17). Sage.

3. Brandhorst, M. (2013). Woran scheitert Kants Theorie der Freiheit? In Brandhorst, M., Hahmann, A., \& Ludwig, B. (Hg.), Sind wir Bürger zweier Welten?: Freiheit und moralische Verantwortung im transzendentalen Idealismus (pp.279-310). Felix Meiner Verlag.

4. Common Curriculum Police Ethics and Prevention of Corruption (2005). Austria: CEPOL

5. Correia, M. G., \& Bleicher, R. E. (2008). Making Connections to Teach Reflection. Michigan Journal of Community Service Learning, 14 (2), 41-49.

6. Gapšyte, R., \& Snieškienè, D. (2015). Ethical Competence of Social Worker and Possibilities for its Development in Practice. Social Work: Experience And Methods, 7 (1), 113-129.

7. Hautzinger, M., \& Pössel, P. (2017). Kognitive Interventionen. Hogrefe Verlag.

8. Lam, C. M., Wong, H., \&Leung, T. F. (2007). An Unfinished Reflexive Journey: Social Work Students' Reflection on their Placement Experiences, British Journal of Social Work, 37 (1), 91-105.

9. Laurinavičius, A. (2001). Administravimo pareigūnu etika. Kaunas, Kau- 
no kolegijos Leidybos centras.

10. Laužackas, R. (1999). Sistemoteorinès profesinio rengimo kaitos dimensijos. Kaunas: VDU leidykla.

11. Lay, K., \& McGuire, L. (2010). Building a Lens for Critical Reflection and Reflexivity in Social Work Education. Social Work Education, 29(5), 539-550.

12. Palidauskaitė J. (2007). Viešojo administravimo etika. Kaunas. Technologija.

13. Rabe, M. (2006). Ethik in der Pflegeausbildung. Ethik in der Medizin, 18(4), 379-384.

14. Richter, S. D., Henkens, C., \& Ritt, S. (2011). Ethische Kompetenz. Organisationsberatung, Supervision, Coaching, 18(4), 413-428.

15. Rutkowsky F. (2017). Wir sind die Guten! Ethik für die Polizei. Verlag: Frank Rutkowsky.

16. Smalskys V. (2008). Policijos personalo rengimo šiuolaikinès kryptys. Viešoji politika ir administravimas, 23, 88-97.

17. Wagener U. (2015). Polizeiliche Berufsethik. Ein Studienbuch. Verlag Deutsche Polizeiliteratur GmbH.

18. Weinert, F. E. (2001). Concept of Competence: A Conceptual Clarification. In Rychen, D. S. \& Salganik, L. H. (Hg.)., Defining and Selecting Key Competencies (pp.45-65). Seattle: Hogrefe\&Huber. 


\title{
ETINESS KOMPETENCIJOS UGDYMAS RENGIANT POLICIJOS PAREIGŪNUS
}

\author{
Dr. Giedrè Paurienė \\ Mykolo Romerio universiteto Viešojo saugumo akademija
}

\section{Santrauka}

Straipsnyje aptariamas policijos pareigūno etinès kompetencijos ugdymas šiuolaikinèje aukštojoje mokykloje. Policija reprezentuoja valstybès autoritetą bendraudama su piliečiais. Savo kasdieneje tarnyboje nuolat kontaktuojama su įvairiais žmonèmis ir, nepaisant šių žmonių skirtingų socialinių pozicijų, policijos pareigūnus jie turètų laikyti patikimais, teisingais ir profesionaliais. Ar vyks sẻkmingas bendravimas su piliečiu, priklauso ne tik nuo situacijos, bet ir nuo policijos pareigūno profesionalumo laipsnio. Kadangi policijos pareigūnai reikalingi sprendžiant ịvairių visuomenès lygmenų konfliktus, etinių dilemų sprendimo gebèjimai turètų tapti profesinès kompetencijos dalimi. Todèl policijos pareigūno etikos kompetencijos, kaip policijos veiklos profesinès kompetencijos sudedamosios dalies, pažinimas, ugdymas, plètotè yra svarbus momentas policijos pareigūnų rengime. Šio darbo tikslas - aptarti etinès kompetencijos ugdymo aukštojoje mokykloje galimybes. Tyrimo objektu pasirinktas policijos pareigūnų etinès kompetencijos ugdymas. Darbe taikomi teoriniai metodai: mokslinių šaltinių analizè, apibendrinimas.

Pirmojoje darbo dalyje atskleidžiamas etinès kompetencijos turinys, t. y. kokiomis etinès kompetencijos struktūros dalimis turètų disponuoti policijos pareigūnas, akcentuojama profesinè deontologija bei svarbiausia deontologinio intuityvizmo kategorija - pareiga, privalejimas. Antrojoje dalyje teigiama, kad suaugusiuju etinès kompetencijos ugdymas dažniausiai vyksta savaiminiu mokymusi; pastebèjus neatitikimą tarp teorijos ir praktikos visada bus teikiamas pirmumas praktinei patirčiai. Prieinama prie išvados, kad policijos pareigūnų etinès kompetencijos ugdymo procesą turi sudaryti sisteminga profesinių moralinių reikalavimų refleksija, kad ugdant etinę kompetenciją yra svarbu patirties reflektavimas ir lyginimas su kitų patirtimi. Taigi ugdant(is) etinę kompetenciją aktualu kurti sąlygas ir reflektavimo kompetencijai bei ją sudarančių gebẻjimų, ign̄̄žių ugdymuisi aukštojoje mokykloje.

Pagrindiniai žodžiai: etinė kompetencija, profesinė deontologija, pareiga, kompetencijos ugdymas(is), refleksija, policijos pareigūnų rengimas. 


\section{AUTORIAUS LYDRAŠTIS}

Autoriaus vardas, pavardė: Giedrè Paurienè

Mokslo laipsnis ir vardas: socialinių mokslų (edukologija) daktarè

Darbo vieta ir pareigos: Mykolo Romerio universiteto Viešojo saugumo akademija, lektoré

Autoriaus mokslinių interesų sritys: profesinės užsienio kalbos mokymo(si) problemos; kompetencija ir savaiminis mokymasis, tarpkultūrinès, etinės kompetencijos plètotè.

Telefonas ir el. pašto adresas: (8 37) 303664; pauriene@mruni.eu

\section{AUTHOR'S COVER LETTER}

Author's name and surname: Giedrè Paurienè

Academic degree and name: Doctor of Social Sciences (Education)

Workplace and position: Lecturer, Public Security Academy, Mykolas Romeris University

Author's research interests: problems of teaching/learning ESP, competence and informal learning, development of intercultural, ethical competence

Telephone and e-mail address: +370 37 303664; pauriene@mruni.eu 